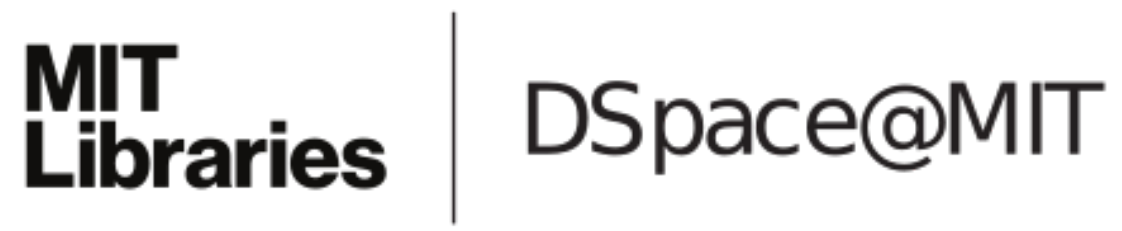

\author{
MIT Open Access Articles
}

Phase-Conjugate Optical Coherence Tomography

The MIT Faculty has made this article openly available. Please share how this access benefits you. Your story matters.

Citation: Le Gouet, J. et al. "Phase-conjugate optical coherence tomography." Lasers and Electro-Optics, 2009 and 2009 Conference on Quantum electronics and Laser Science Conference. CLEO/QELS 2009. Conference on. 2009. 1-2. (C2009 Institute of Electrical and Electronics Engineers.

Publisher: Institute of Electrical and Electronics Engineers

Persistent URL: http://hdl.handle.net/1721.1/59827

Version: Final published version: final published article, as it appeared in a journal, conference proceedings, or other formally published context

Terms of Use: Article is made available in accordance with the publisher's policy and may be subject to US copyright law. Please refer to the publisher's site for terms of use. 


\title{
Phase-Conjugate Optical Coherence Tomography
}

\author{
J. Le Gouët, D. Venkatraman, F. N. C. Wong and J. H. Shapiro \\ Research Laboratory of Electronics, Massachusetts Institute of Technology, Cambridge, MA, USA \\ le_gouet@mit.edu
}

\begin{abstract}
We demonstrate a new type of optical coherence tomography using only classical resources to achieve results that are typically associated with quantum-enhanced metrology: factor-oftwo axial resolution enhancement and even-order dispersion cancellation.

(c) 2009 Optical Society of America
\end{abstract}

OCIS codes: (120.3180) Interferometry; (270.5585) Quantum information and processing

\section{Introduction}

The use of quantum resources, such as entanglement, to enhance measurement capabilities is of great interest in metrology and interferometry. For example, entangled photon pairs from spontaneous parametric downconversion (SPDC) have been utilized in quantum-optical coherence tomography (Q-OCT) [1] to demonstrate an axial resolution improvement of a factor of two with even-order dispersion cancellation. Recent research suggests that in some cases the enhanced measurement sensitivities are not due to entanglement, and therefore can in principle be replicated using classical resources. Such classical techniques shed light on the fundamental basis of the measurement enhancement and may offer significant advantages in signal-to-noise ratios (SNR) and acquisition time over methods using quantum resources, because the latter are often difficult to generate, manipulate and detect efficiently.

In the case of Q-OCT, Erkmen and Shapiro [2] have shown that its measurement advantages do not derive from entanglement but rather from the phase-sensitive cross correlation between the signal and idler. Moreover, based on this discovery, they proposed a new technique, called phase-conjugate optical coherence tomography (PC-OCT) [2], that uses classical resources with an unconventional but still classical receiver to achieve the same factor-of-two resolution enhancement and even-order dispersion cancellation as Q-OCT. In this work, we report the experimental realization of PC-OCT demonstrating these Q-OCT advantages, but with an SNR that is orders of magnitude higher.

\section{PC-OCT experimental setup}

Figure 1 shows the PC-OCT experimental setup. We chose amplified type-II SPDC for the reference and conjugate probe beams, thus providing the maximum, classically allowable phase-sensitive cross correlation. The parametric fluorescence was generated by pumping a $2-\mathrm{cm}$ periodically poled $\mathrm{MgO}$-doped lithium niobate (PP-MgO:LN) crystal with 50-ps pulses ( $2 \mathrm{~W}$ average power at $31 \mathrm{MHz}$ repetition rate) at $780 \mathrm{~nm}$. We optimized the emission of this broadband source ( $120 \mathrm{~nm}$ bandwidth) to get $70 \%$ of the total SPDC power coupled into a single-mode fiber, yielding at the output a spectral brightness of more than $1 \mathrm{nW} / \mathrm{nm}$. A motorized delay line was used to match the path lengths of the reference and probe arms. Both beams were filtered with a bandwidth of $0.4 \mathrm{~nm}$ or $5 \mathrm{~nm}$.

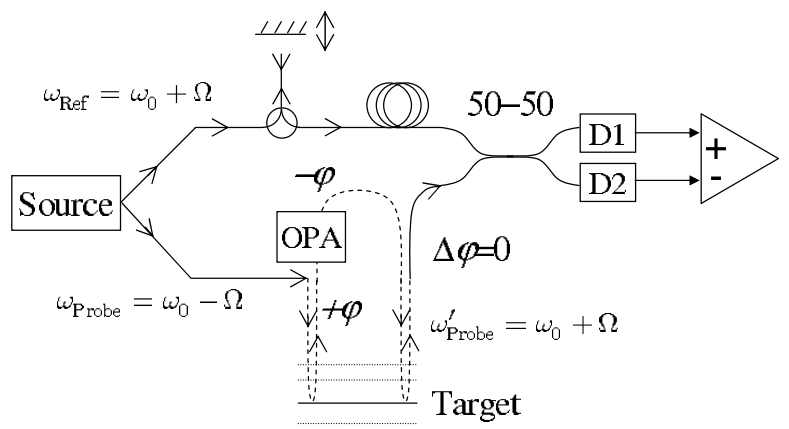

Fig. 1. PC-OCT experimental setup. The dashed (resp. solid) line represents the free-space (resp. fiber coupled) path.

The target, a high-reflector mirror on a motorized translation stage, was sampled twice, first by the 1570-nm probe beam and again by the $1550-\mathrm{nm}$ conjugate beam produced by an optical parametric amplifier (OPA) with the returned probe beam as its input. The probe and conjugate beams were phase-anticorrelated such that even-order 


\section{IPDB7.pdf}

dispersion along their common path could be canceled. The OPA had a maximum gain of $20 \mathrm{~dB}$, to compensate for target transmission losses. The returned conjugate beam was combined with the reference beam at a 50-50 fiber beam splitter, and their interference was detected with high-sensitivity InGaAs photodetectors. The distinct features of PC-OCT are the use of a high-flux source with phase-sensitive cross correlation, target sampling in a double-pass configuration, and parametric amplification to provide gain and phase conjugation.

\section{Axial resolution enhancement and dispersion cancellation}

The axial resolution measurement was first made with 10 -ps pulses with a spatial coherence length of $3 \mathrm{~mm}(0.4-\mathrm{nm}$ bandwidth), so that dispersion was not significant. We measured the amplitude of the reference-conjugate interference envelope as a function of the position of the reference-arm delay stage. Each of the two curves in Fig. 2(a) shows a spatial full width at half maximum (FWHM) $\Delta z_{\mathrm{FWHM}}$ of $2.36 \mathrm{~mm}$, in very good agreement with the calculated correlation of two 10-ps pulses.

To illustrate the two-fold improvement in axial resolution, we measured the interference envelope for two successive target positions. Figure 2(a) shows that a reference delay shift $\Delta z_{\text {Ref }}$ of $2.22 \mathrm{~mm}$ was applied to recover the maximum contrast for a target positional shift $\Delta z_{\text {Target }}$ of $1.18 \mathrm{~mm}$, corresponding to half of $\Delta z_{\text {Ref }}$ with a relative error of $6 \%$ (due to the thermal fluctuations of the reference fiber length). In comparison, we have also set up a standard single-pass OCT configuration and the same reference delay shift yielded a target positional shift $\Delta z_{\text {Target }} e q u a l$ to $\Delta z_{\text {Ref }}$ of $2.38 \mathrm{~mm}$. In other words, the results show that the double-pass configuration resolves target separations $\Delta z_{\text {Target }}$ that are half of $\Delta z_{\mathrm{FWHM}}$, thus increasing the axial resolution by a factor of two.
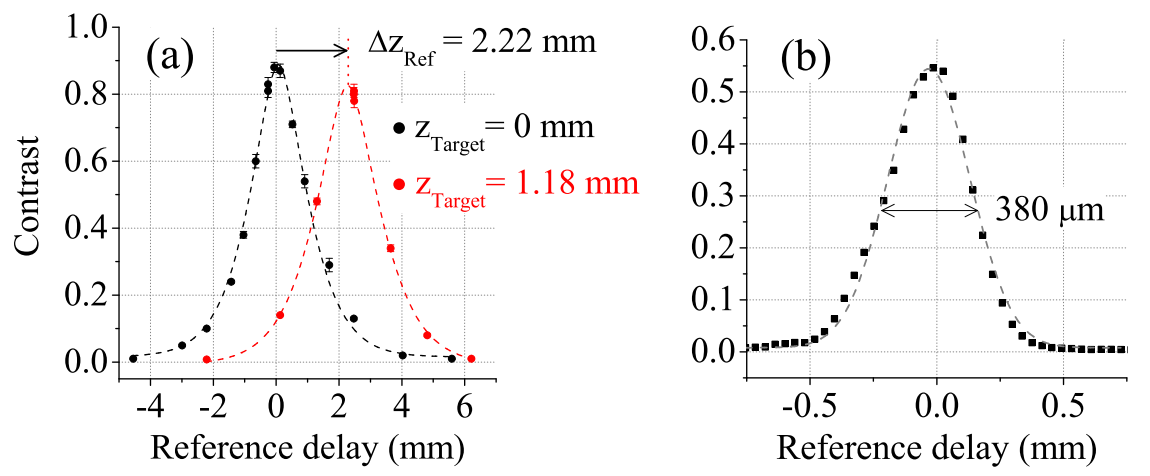

Fig. 2. (a) Data (points) and theoretical model (dashed line) for PC-OCT measurement, with 10-ps reference and probe pulses. The two curves represent the interference contrast as a function of the reference path delay for two positions of the target separated by 1.18 mm. (b) Dispersion cancellation measurement using 0.8-ps pulses yields a resolution of $380 \mu \mathrm{m}$, compared with $780 \mu \mathrm{m}$ if dispersion were not canceled.

To confirm the dispersion cancellation capability of PC-OCT, we changed the spectral filter to a $5 \mathrm{~nm}$ bandwidth, corresponding to a $\sim 0.8$ ps pulse width, or a spatial coherence length of $0.24 \mathrm{~mm}$. The probe arm had 51 meters of SMF28 fiber, with a dispersion value of $\sim 17 \mathrm{ps} / \mathrm{km} / \mathrm{nm}$. Dispersion cancellation occurred for the length of fiber within the double-pass configuration of the probe arm. Because dispersion could not be corrected in the reference arm, we used a dispersion-shifted fiber wherever possible, comprising 37 meters of such fiber (4 ps/km/nm). Figure 2(b) shows a typical interferometric envelope with a measured width $\Delta z_{\mathrm{FWHM}}$ of $380 \mu \mathrm{m}$. This is in good agreement with our model, taking into account the dispersion in the reference arm and the small uncompensated section in the probe arm. As a comparison, we estimate that if dispersion cancellation were absent, the measured width would be $\sim 780 \mu$ m. Note that the reduced $\Delta z_{\mathrm{FWHM}}$ is entirely due to the larger spectral bandwidth used in the dispersion measurements.

In summary, we have demonstrated for the first time the new technique of phase-conjugate optical coherence tomography, showing the factor-of-two axial resolution enhancement, along with dispersion cancellation. We used only classical resources, including amplifiers and standard photodetectors to achieve the same level of measurement sensitivity as obtained by quantum-optical coherence tomography, with one significant advantage: the SNR was orders of magnitude higher, allowing data acquisition rates of less than a second per data point.

\section{References}

1. M.B. Nasr, B.E.A Saleh, A.V. Sergienko, and M.C. Teich, "Demonstration of dispersion-canceled quantum-optical coherence tomography," Phys. Rev. Lett. 91, 083601 (2003).

2. B.I. Erkmen and J.H. Shapiro, "Phase-conjugate optical coherence tomography," Phys. Rev. A 74, 041601(R) (2006). 